\title{
Heating Dynamics Simulation of H274Y Mutant Neuraminidase-Inhibitors Complexes
}

\author{
Sigit J. Herlambang and Rosari Saleh
}

\begin{abstract}
The H274Y mutant neuraminidase, known as oseltamivir-resistant, can be inhibited by zanamivir and laninamivir. The inhibition of oseltamivir-resistant neuraminidase has been experimented in vivo and in vitro in several experiments. To complete the usage of zanamivir and laninamivir inhibition experiments, we investigate the structural and dynamics of neuraminidase-inhibitors complexes using heating dynamics simulation. All processes which support the simulation also reported to produce a vast review of the subject. Through homology modeling method and models assessments, the best model (model number 3) was selected to proceed to the attachment of the substrate via CDOCKER molecular docking. The best docked pose was continued to energy minimization and heating dynamics simulation. The simulation results have been observed using root mean square deviation (RMSD), the percentage of hydrogen bonds occupancy, and the binding free energies comparison. From these results, we have concluded that the high value of the binding free energies, the value and the fluctuation of neuraminidase-oseltamivir RMSD, and the percentage of hydrogen bonds occupancy may be related with the resistance of H274Y mutant neuraminidase to oseltamivir. The simulation results were also correlated to the success of laninamivir and zanamivir to bind $\mathrm{H274Y}$ mutant neuraminidase compared with peramivir and oseltamivir. Both laninamivir and zanamivir are preferable to treat $\mathrm{H} 274 \mathrm{Y}$ mutant neuraminidase that could resist oseltamivir carboxylate.
\end{abstract}

Index Terms-Influenza, neuraminidase, mutant, simulation

\section{INTRODUCTION}

Continuous outbreaks of $\mathrm{H} 5 \mathrm{~N} 1$ avian influenza that able to infect humans has been happening for the last 16 years. Recent event of human infection was reported to the World Health Organization (WHO) in January, $24^{\text {th }} 2012$ [1]. Since 1996, the first recorded event of highly pathogenic H5N1 virus, many countries with the economical background in poultry farming have been struggling from the avian flu invasion and its localized outbreaks. Indonesia, one of the countries mentioned before, is also impacted by these potential threats. The new strain $\mathrm{H} 5 \mathrm{~N} 1$ virus is known has been spreading over poultries in 31 of 33 provinces and 12 of them were reported hundreds cases of human infection [2]. As of March, $5^{\text {th }} 2012,186$ cases occurred in Indonesia with 154 deaths have been reported in cumulative number of confirmed human cases for avian influenza A H5N1 reported to WHO during 2003-2012 [3]. Today, Indonesia becomes the country with the most human deaths surpassing Vietnam

Manuscript received March 31, 2012; revised May 12, 2012

The authors are with the Departemen Fisika, Fmipa, Universitas Indonesia, P.O. Box 16424 Indonesia (e-mail: sigit.jaya.herlambang@gmail.com, sigit.jaya.herlambang@r103a.com). and Egypt.

The next, mortal, and larger outbreak which could become an epidemic is possible to happen someday if there is no pressure acts and prevention for $\mathrm{H} 5 \mathrm{~N} 1$ virus transmission. Thus, the routine and continued surveillance is needed in several places known as the potentially infected places such as poultry farms and markets. Ortrud Werner and Timm C Harder wrote in the influenza report that, over the years, the increment of virulence of $\mathrm{H} 5 \mathrm{~N} 1$ for mammals and the expansion of host range are keep growing [4]. It also had been reported that in China, from 1999 to 2002, and in Vietnam since 2003, the H5N1 virus has become progressively more pathogenic for mammals [5]. Further review from Georg Behrens and Matthias Stoll defined two qualities of influenza account for much of the epidemiological spread of the virus. Include the ability to emerge and circulate in avian or porcine reservoirs by either genetic re-assortment or direct transmission and subsequently spread to humans at irregular intervals, and the fast and unpredictable antigenic change (drift and shift) of important immune targets once the virus have become established in a human [6]. Therefore, the knowledge related with transmission process is become the key to prevent global spread of H5N1 [7].

Both hemaglutinin (HA) and neuraminidase (NA), are known to play an important role in replication and transmission process [8]. Penetration of the virus into the host cell requires HA molecule to bind the sialic acid (SA). The detachment of matured virion from the host cell utilize NA molecule to cleave SA so that the virus is able to infect other host cells. A single mutation in one of these two glycoproteins is able to initiate the resistance against inhibitors. A few specific NA residual mutations in neuraminidase, such as R292K, H274Y, R152K, E119V, and N294S, have created a huge impact in tamiflu treatment (commercial name of oseltamivir) [9]-[19]. Further studies on the oseltamivir-resistant correlated with H274Y mutant had been reported due to the reorientation of the adjacent E276 residue forcing its carboxyl group to move closer to the binding site [9], [20]. A number of experiments have responded this issue with alternative compounds as a promising competitor of SA binding with the H274Y mutant NA [21]-[23]. They proposed laninamivir and zanamivir to resolve the issue of oseltamivir resistance.

The main objective of this study is to simulate neuraminidase-inhibitors complexes and compare the interactions between them during the heating dynamics simulation. To achieve this, a series of sequence alignment, phylogenetics, homology modeling, protein assessments, molecular docking, and structural energy minimization had been completed before being followed with heating dynamics 
simulation. The structural and energetic investigation, such as RMSD value and fluctuation, the hydrogen bonds occupation, and the binding free energies, had been collected from molecules trajectories post-simulation. We realize that the simulation is way too short to reveal the conformational changes of the complex molecules. But in this time interval, we are able to observe the atoms in the active site and their atomic interaction with inhibitors.

\section{MATERIALS AND METHODS}

\section{A. Molecules Preparation}

The National Center of Biotechnology Information (NCBI) influenza database contained with many proteins and nucleotides sequences [24]. It is including H274Y mutant NA type 1 (N1) sequence from the high pathogenic avian influenza virus (A/Indonesia/560H/2006(H5N1)) which has been stored with the accession code ABW06159. In NCBI influenza database, we also found another 133 NA sequences collected around the years 2003-2007, but sequence which has H274Y mutation is only ABW06159. The collected protein sequence, contained with 449 amino acids, was used to identify homologous template molecule. The template identification had been done using BLASTP 2.2.16 [25] and InterProScan [26] provided by Swiss Expasy [27]-[31]. The closest homologous template, with the greatest percentage of identity, suggested from Swiss Expasy template identification is a crystal structure with protein data bank code 3CKZ. The molecule of 3CKZ was taken from Research Collaboratory for Structural Bioinformatics (RCSB) [32].

TABLE I: THE ACCESSION CODES OF NA'S OF INFLUENZA VIRUSES FROM PANDEMICS OCCURRED DURING THE $20^{\text {TH }}$ AND $21^{\text {ST }}$ CENTURIES

\begin{tabular}{|l|l|l|l|}
\hline No. & $\begin{array}{l}\text { Accession } \\
\text { Code \& } \\
\text { Sequence } \\
\text { Name }\end{array}$ & Virus Name & Note \\
\hline 1. & AAF77036 & $\begin{array}{l}\text { A/Brevig } \\
\text { Mission/1/1918(H1N1) }\end{array}$ & $\begin{array}{l}\text { Obtained at } \\
\text { Spanish flu } \\
\text { occurred }\end{array}$ \\
\hline 2. & AAT66420 & A/Japan/305/1957(H2N2) & $\begin{array}{l}\text { Obtained asian flu } \\
\text { Ascurred } \\
\text { occur }\end{array}$ \\
\hline 3. & AAO46476 & $\begin{array}{l}\text { A/England/878/1969(H3 } \\
\text { N2) }\end{array}$ & $\begin{array}{l}\text { Obtained at } \\
\text { occurred flu }\end{array}$ \\
\hline 5. & ADL39249 & $\begin{array}{l}\text { A/Alaska/WRAIR1114P/ } \\
\text { 2009(H1N1) }\end{array}$ & $\begin{array}{l}\text { Obtained at } \\
\text { 2009 } \\
\text { pandemic }\end{array}$ \\
\hline 5. & 3 CKZ & $\begin{array}{l}\text { Sequence of } \\
\text { template } \\
\text { structure }\end{array}$ \\
\hline
\end{tabular}

The sequence has been aligned with a few NA sequences. Those sequences had been extracted from the virus which had been spread out during prevalent pandemics/infections, and the chosen template sequence (see Table I). Those NAs were analyzed using multiple sequences alignment with parameters focused on the consensus and trace residues to see the similar pattern of the viruses. A phylogenetic tree has been produced using parameters neighbor joining method [33], the best tree mode and set a distance as Uncorrected ('p'). Those parameters have been set to simplify the analysis because the results were given as percentages of different residues within aligned sequences.

The models created by homology modeling using MODELER [34]. The medium optimization level and cut overhangs have been set. The cut overhangs addition is able to remove the terminal of unaligned residues in models. The homology modeling generated five models from the template. The models are then evaluated using three different protein assessments. The Discrete Optimized Protein Energy (DOPE) method [35] and 3D-profiles [36], [37] were used to verify models stability and reliability. The DOPE-HR method was conducted to models evaluation in MODELER. It is similar with regular DOPE method, but obtained at higher resolution. The smooth windows sized 10 and Kabsch-Sander algorithm for secondary structure was set for 3D-profiles method [38]. Besides DOPE scoring and 3D-profiles, all models also sent to Swiss Expasy to be analyzed with PROCHECK [39]-[41] to get insight the stereo chemical properties of the models using Ramachandran analysis.

\section{B. Molecular Docking, Minimization, and Heating Dynamics Simulation}

TABLE II: LIST OF INHIBITOR COMPOUNDS USED IN THIS STUDY AND THEIR

\begin{tabular}{|l|l|l|l|}
\hline Figure & ID & Name & Formula \\
\hline & & Zanamivir & $\mathrm{C}_{12} \mathrm{H}_{20} \mathrm{~N}_{4} \mathrm{O}_{7}$ \\
\hline & & & \\
\hline & & Peramivir & \\
\hline & & & \\
\hline
\end{tabular}

The compounds and substances database PubChem provides over 31 million compounds, 75 million substances and bioactivity results from 1644 high-throughput screening programs [42]. The structures of inhibitors listed in Table II were collected from PubChem chemical molecules database, which is maintained by NCBI. Actually, there are 13 neuraminidase inhibitor compounds, but only four inhibitors had been used in this study. The selection of these neuraminidase inhibitors was based on the experiment of Kiso et al. which used laninamivir, zanamivir, peramivir and oseltamivir carboxylate [23].

The best neuraminidase model is selected according to the value of DOPE method, 3D-profile, and PROCHECK. Then, the model is attached with peramivir, laninamivir, zanamivir, 
and oseltamivir carboxylate one by one using CDOCKER [43]. Ten random top hits and conformations, 1000 random conformations dynamics steps and conformations dynamics target temperature, including electrostatic interactions and orientation van der Waals energy threshold, and use CHARMm [44]-[45] for forcefield and ligand partial charge were determined for the parameters used. The candidate poses were created using random rigid-body with six degrees of freedom (three rotations/three translations) followed by simulated annealing. The sphere was generated to fill the active site. The generation of the sphere is to facilitate ligand matching in the sphere centre and to find possible ligand orientations. Following that, a final minimization used to refine the ligand position in the active site. A pose with minimum interaction energy would be continued to the next energy minimization and heating dynamics simulation.

All best pose molecules were subjected to two steps of energy minimization to produce an optimum geometrical structure. The first step was executed by the steepest descent with a targeted energy gradient of $0.5 \mathrm{kcal} /(\mathrm{mol} \mathrm{x} \AA$ ) and a $1,000,000$ steps maximum. The second step was executed by the conjugate gradient with a targeted energy gradient of 0.1 $\mathrm{kcal} /(\mathrm{mol} \mathrm{x} \AA)$ and a $1,000,000$ steps maximum. The Generalized Born Molecular Volume (GBMV) implicit solvent energy to mimicking the aqueous environment [46], SHAKE algorithm [47], a non bond list radius of $14 \AA$, and a switching function was applied between 10-12 $\AA$ for a computational efficiency [48]-[49]. To gain a long-range electrostatic energy contribution, it was visualized in a spherical cutoff mode.

A 100 picosecond (ps) time simulation was conducted to all optimized structures and rising the system temperature from 0 into $300 \mathrm{~K}$ with 50,000 steps, two femtoseconds (fs) time step, and the trajectory data were stored every $0.1 \mathrm{ps}$. Other parameters such as GBMV implicit solvent, SHAKE algorithm, non bond list radius, and switching function were determined identical with minimization energy set up.

After the heating dynamics simulation, the binding free energies were calculated in vacuum environment which constructed from the each molecule average Gibbs energy. The temperature and entropy contribution were included in the calculation and combined with molecular mechanic energy contribution ( $\bar{E}_{M M}$ ), which is the energy term produced by the applied forcefield and also the potential energy of the system [50]:

$$
E_{M M}=E_{\text {potential }}=E_{\text {bond }}+E_{\text {angle }}+E_{\text {torsion }}+E_{\text {outofplane }}+E_{\text {electrostaic }}+E_{\text {vanderWaas }}
$$

The relationship between the ligand, receptor, and complex energy is given in the next equation:

$$
\Delta \bar{G}_{\text {binding }}=\bar{G}_{\text {complexl }}-\left(\bar{G}_{\text {ligand }}+\bar{G}_{\text {receptor }}\right)
$$

The average Gibbs energy which constructed each component energy in the (2) equation is the binding free energy of the complex. Furthermore, all phases of the study described in this section from structure preparation to molecular dynamics simulation were conducted with Discovery Studio 2.1 (Accelrys).

\section{RESULTS}

\section{A. Molecules Preparation}
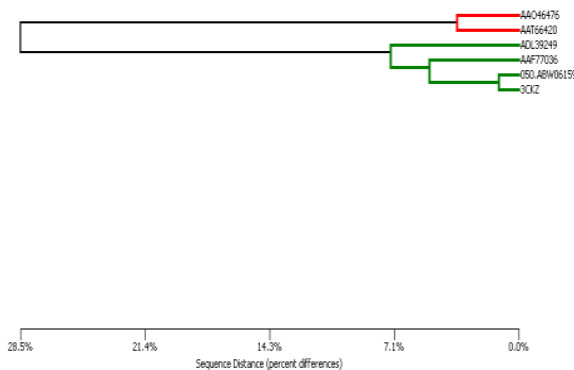

Fig. 1. The phylogenetic tree of ABW06159 correlated with several viruses.

The evolution link is shown in Fig. 1 as a phylogenetic tree. Template 3CKZ was identified have a 97\% similarity, which is the most similar sequence, with ABW06159 sequence. Another NA sequences which have closer relationship with ABW06159 sequence are the sequences obtained from 1918s epidemic and 2009 pandemic. The differences between ABW06159 and 2009 pandemic is higher than 1918s epidemic. Different types of NA were observed from Asian and Hongkong flu pandemics. The differences between ABW06159 with both of them are up to $28 \%$.

Five models have been generated by Build Homology Modeling from 3CKZ (homologous template) and shown in Fig. 2. Refinement and codon renumbering were subjected to all models. The reason of models refinement be caused that the $3 \mathrm{CKZ}$ template has a few broken peptide bonds and repeated numbers such as 169 and 169-A residues, 345 and 345-A, and 412, 412-A, 412-B, 412-C and 412-D. Those repeated numbers does not mean that there are two residues in one position, but only the next residue has numbered, with the same number and the alphabetical addition after the number, as its previous position residue.

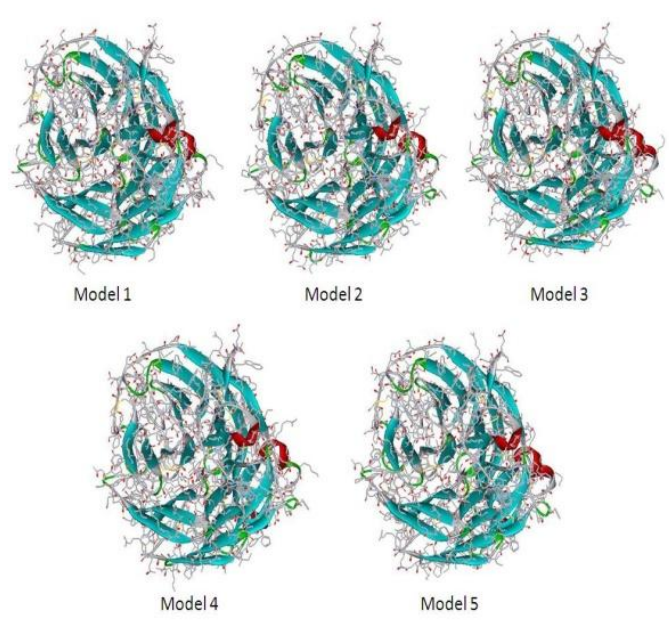

Fig. 2. Five models of H274Y mutant ABW06159.

The models and template structures were assessed using three different methods. The results of protein structures assessment are shown in the Table III. The verify scores of all models vary from 192-201, which is higher than the high expected score, 175.54. The highest score was occupied by model 3, which being the only one model exceeded template verify score. 
Second assessment was executed using DOPE-HR scores. The DOPE-HR score relate with the stability of molecules. The higher DOPE-HR score indicated the worst model. All DOPE score of the structures assessed vary in range -36386 to -34793 . The lowest DOPE-HR score of structures assessed is template $3 \mathrm{CKZ}$. The comparison without template show that model 3, which have the lowest DOPE-HR score compared than other models.

PROCHECK protein assessment revealed the stereo chemical properties of structures. The result from PROCHECK provides ramachandran plots of all NA residues without glycine and proline. In the Table III, the PROCHECK data shown is the percentage of NA residues which in the most favoured and additionally allowed regions which have been extracted from ramachandran plots. The template is taking the first place with $99.7 \%$ followed by model 3 and 4 with $99.4 \%$.

All assessment methods being used in this experiment are in agreement that the model 3 is the best NA model compared with other models generated.

\section{B. Molecular Docking, Minimization, and Heating Dynamics Simulation}

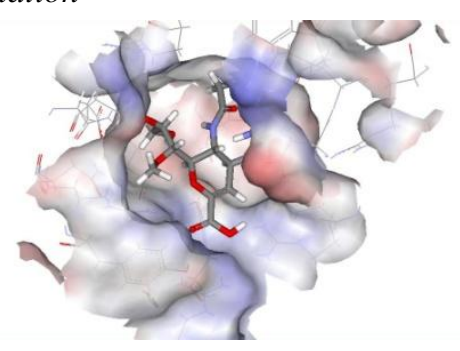

a

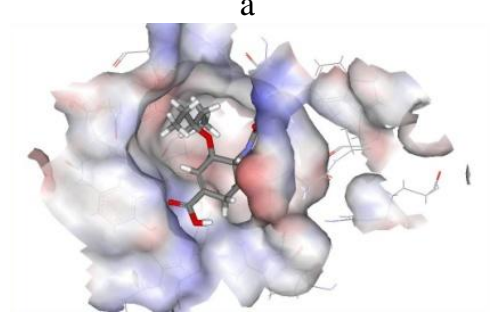

b

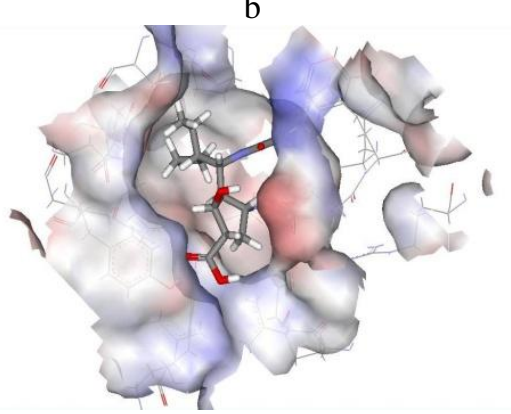

c

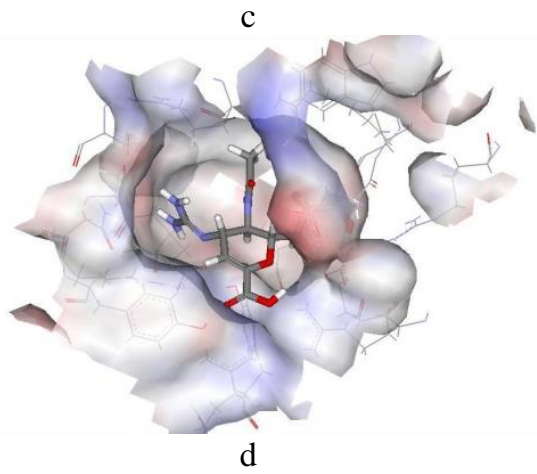

Fig. 3. The best pose structure of: a. NA-LA complex; b. NA-OS complex; c. NA-PE complex; d. NA-ZA complex.
TABLE III: THE ASSESSMENT SCORES OF FIVE HOMOLOGY MODELS

\begin{tabular}{|c|c|c|c|c|}
\hline $\begin{array}{l}\text { Template } \\
\text { and Models }\end{array}$ & $\begin{array}{l}\text { Verify } \\
\text { Score }\end{array}$ & DOPE & $\begin{array}{l}\text { Most } \\
\text { Additional } \\
\text { Percentage } \\
\text { and Pro) }\end{array}$ & $\begin{array}{r}\text { Favoured\& } \\
\text { Residues } \\
\text { (without Gly }\end{array}$ \\
\hline Model 1 & 192.99 & -35183 & $98.8 \%$ & \\
\hline Model 2 & 198.73 & -34793 & $99.1 \%$ & \\
\hline Model 3 & 200.3 & -36386 & $99.4 \%$ & \\
\hline Model 4 & 197.4 & -35510 & $99.4 \%$ & \\
\hline Model 5 & 197.98 & -35311 & $99.1 \%$ & \\
\hline $\begin{array}{l}\text { Template } \\
3 \mathrm{CKZ}\end{array}$ & 199.53 & -42594 & $99.7 \%$ & \\
\hline
\end{tabular}

The best model, model 3, was subjected to molecular docking with four inhibitors mentioned in Table II. Each NA-inhibitor molecular docking process has conferred 10 poses. The smallest CDOCKER energy, which related with the inreaction, has been used to help selecting the best pose. The best pose was executed further to create a complex molecule. All complexes are shown in Fig. 3a, 3b, 3c, and 3d. The red region in the electrostatic surface of neuraminidase is the negative charges of aspartic acid side chain. After being docked, steepest descent and conjugate gradient energy minimization were executed.

The hydrogen bonds formed in the NA-inhibitor interaction site before and after minimizations were observed. We have found that in NA-ZA and NA-LA complexes, the number of hydrogen bonds formed had increased. In NA-ZA complex, the numbers of the hydrogen bonds formed increase from 11 to 13 whereas in NA-LA is from nine to ten. It is in contrast with NA-peramivir (NA-PE) complex, which shown the decreased number of hydrogen bonds formed from seven to six. The NA-oseltamivir carboxylate (NA-OS) complex shows no change in the number of hydrogen bonds formed with six hydrogen bonds. However, the minimization process resulting in satisfied energy gradient of all complex structures and brought all complexes total energies into -20135.62, -20148.52, -20142.29, and -20138.44 kcal/mol, for NA-LA, NA-OS, NA-PE, and NA-ZA, respectively.

The heating dynamics simulation has been done to check the stability and the interaction of structures during the simulation. Heating dynamics simulation increases the temperature of the system from 0 into $300 \mathrm{~K}$. The system was heated from 0 to $300 \mathrm{~K}$ in the first $2.5 \mathrm{ps}$ then fluctuating in the range 296-305 K. The total energies of NA-LA, NA-OS, NA-PE, and NA-ZA complexes rose as much as kinetic energies. The trajectories stored were analyzed to get insight the NA-inhibitors interaction. The trajectories contained with the data of the simulated molecule complexes. 
The binding free energy is able determine the ability of enzyme protein to bind its substrate [51]-[52]. In this simulation, the binding free energies were calculated in vacuum through all trajectories recorded. The averages of data from each complex were listed in Table IV. The results show that NA-ZA and NA-LA complexes have lower binding free energies than NA-OS and NA-PE complexes. This is in accordance with other results in this current study that oseltamivir and peramivir have less strength in binding with H274Y mutant neuraminidase.

TABLE IV: THE BINDING FREE ENERGIES OF NA-INHIBITOR COMPLEXES

\begin{tabular}{|c|c|c|c|}
\hline \multicolumn{4}{|c|}{ Binding Free Energies in Vacuum (kcal/mol) } \\
\hline NA-LA & NA-OS & NA-PE & NA-ZA \\
\hline-215.67 & -103.65 & -116.56 & -239.34 \\
\hline
\end{tabular}

\section{Structural Analysis}

The stability of inhibitors has been monitored during the simulation. The movement of the inhibitors in the active site of NA can be represented by its root mean square deviation (RMSD). The RMSD of four inhibitors were plotted in Fig. 4. The RMSD of all inhibitors show that the movement of oseltamivir carboxylate in the active site is relatively high (showed in the red line). The fluctuation of RMSD almost surpassing $3.5 \AA$ and being the highest value compared with laninamivir, zanamivir, and peramivir. The zanamivir RMSD fluctuation is higher than peramivir. Further observation in the structure of NA-PE complex resulted that peramivir has moving away from NA active site. The number of hydrogen bonds between NA-PE was known decreased after minimization energy had been executed. Because of that, since the peramivir is already far from the active site, the RMSD of it is not really significant. In other hand, the laninamivir has the lowest RMSD and less fluctuated during the simulation. Laninamivir has RMSD fluctuates below $1 \AA$.

The hydrogen bond is able to indicate the strength of the interaction. The better interaction will results in more hydrogen bonds created between them. The strength of hydrogen bond had been monitored from its occupancy in every conformation in the trajectories. Shu et al. has showed that the strong hydrogen bond have a percentage of hydrogen bond occupancy larger than $80 \%$ [53]. In Fig. 5a, 5b, 5c, and $5 \mathrm{~d}$, have been plotted the percentage of hydrogen bond occupancy between NA and its inhibitors during the simulation. The results will be discussed based on Stoll et al. characterization with the addition of E277 into S5 [54].

The hydrogen bond occupancy of NA-LA is shown in Fig. 5a. There are eight NA residues which produced strong hydrogen bonds with laninamivir. In the S1 subsite, the hydrogen bond occupation was dominated by R292. Other residues, R118 and R371, did not giving a big contribution into NA-LA binding. Even R118 does not make any hydogen bond during simulation. The most contribution of bindings is come from S3 and S5 subsites residues. They are W178, S179, S246, E276, and E277 which produced the percentage of occupancy above $80 \%$.

The percentage of hydrogen bond occupancy shown in the Fig. $5 b$ revealed the lack of interaction between NA-OS. All functional residues have a weak interaction during simulation because all of hydrogen bond occupation percentages are below $80 \%$. There are only two residues from S2 subsite, E119 and E227, which almost 80\%. Even the NA-PE interaction is better than NA-OS. It could be observed from the three strong hydrogen bonds formed during the simulation as shown in Fig. 5c. In NA-PE interaction, the hydrogen bond bindings were dominated by D151, W178, and R292.

In Fig. 5d, the interaction between NA-ZA was revealed. The percentage of hydrogen bond above $80 \%$ were made by $\mathrm{S} 1, \mathrm{~S} 3$ and S5 subsites residues such as R118, R152, E276, E277, R292, and catalytic residue D151. In the S1, similar with the interaction between NA-LA, R371 did not give much contribution to the bindings. Alternate binding in the S3 subsite, a hydrogen bond formed by R152, the interaction in S5 subsite of NA-ZA complex have a similarity with what happened in the NA-LA complex, the E276 and E277 have a good interaction with zanamivir.

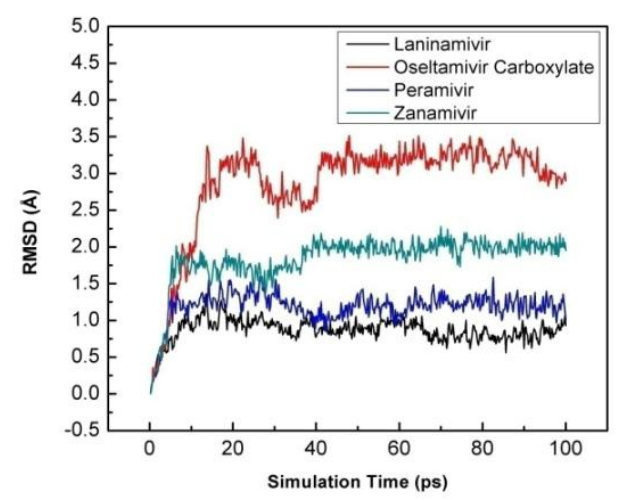

Fig. 4. The RMSD of four inhibitors during the simulation
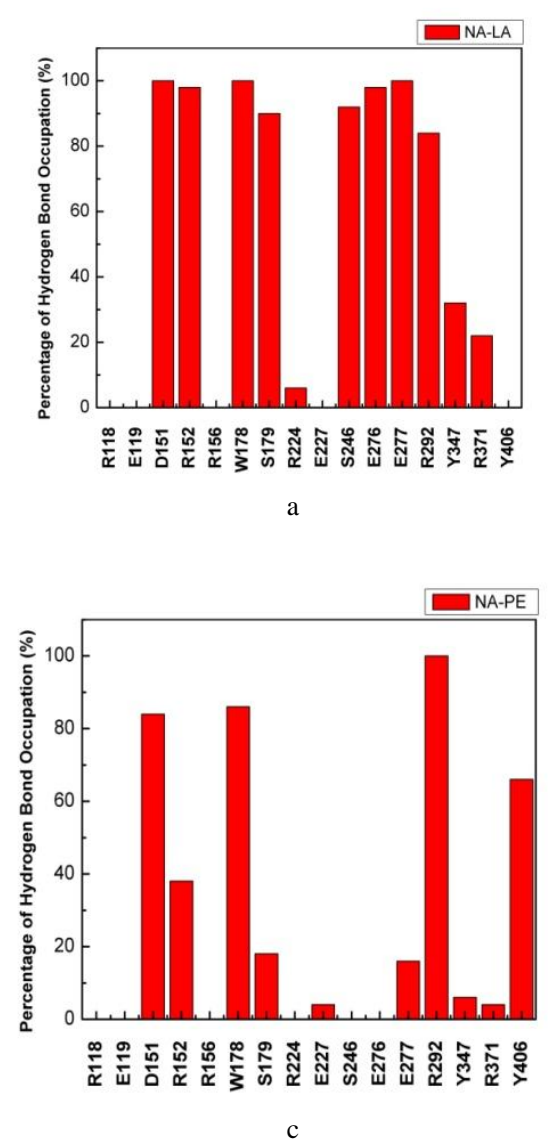


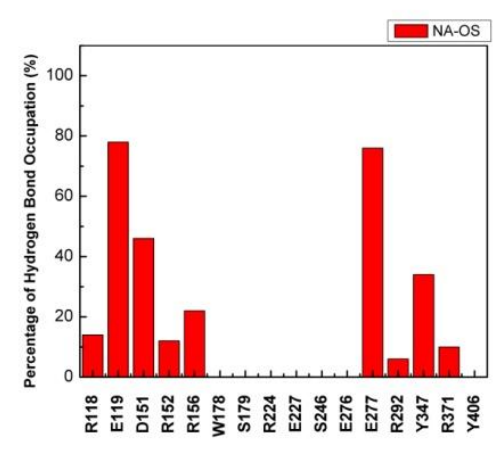

$\mathrm{b}$

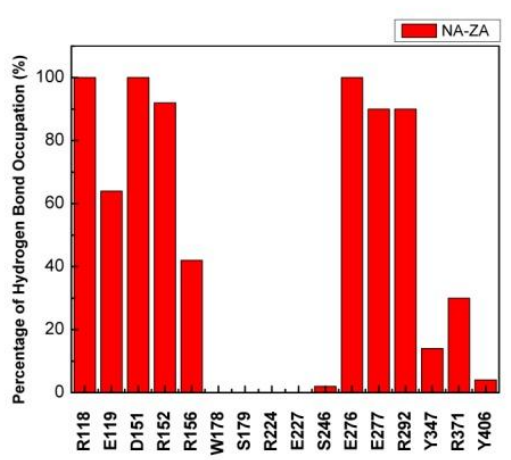

$\mathrm{d}$

Fig. 5. The percentage of hydrogen bond occupancy of: a. NA-LA complex; b. NA-OS complex; c. NA-PE complex; d. NA-ZA complex.

\section{RESULTS}

The aim of this study is to check the stability and the interaction of $\mathrm{H} 274 \mathrm{Y}$ mutant neuraminidase. In order to achieve that, the heating dynamics simulation was subjected to the NA-Inhibitor complexes. All the processes within the series of three different assessment methods, molecular docking, minimization energy, and heating dynamics simulation, had been included in this paper since each of them influences other process. The combination of three different assessment used give a comprehensive approaching to select the best model since every method used has its own specialties. Molecular docking is able to attach inhibitors into the NA enzyme. The minimization energy could optimize the molecule structure before simulated. And the last but not least, the heating dynamics simulation could be used in depth analyses of structure, energy, and electrostatic interaction of the inhibitors with the NA.

The inhibitor response can be compared from its movement, which is represented from RMSD. The highest movement is made by oseltamivir carboxylate whereas the smallest is laninamivir. As far as RMSD comparison is concerned, the findings acquired here is in line with multiple studies that infers how higher substrate RMSD suggest superior NA ability to reject an inhibitor [53], [55]-[56]. Thus, the Indonesian H274Y mutant NA could be said as one of many oseltamivir-resistant.

A few differences in the hydrogen bond contribution between each inhibitor have been observed. The carboxylic group that acts as a "main attraction", particularly for the 118-292-371 triad that initiates binding, is observed to have two residues, R118 and R292, with strong hydrogen bonds only happens in NA-ZA [57]. In contrast to that, NA-LA and NA-PE only had one residue, R292, which has formed strong hydrogen bonds for the exact same region whereas in NA-OS no residue of this $\mathrm{S} 1$ subsite that formed a strong hydrogen bonds. The strong hydrogen bond at Arg292 emphasizes the importance of this residue in NA-inhibitors binding. There are studies that describe inhibitor resistance due to the R292K mutation [09]-[11].

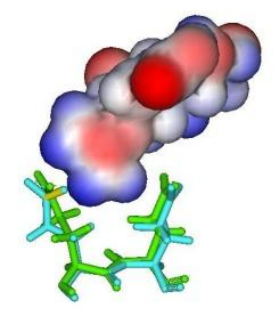

(a)

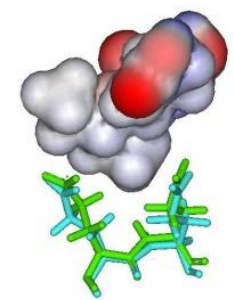

(c)

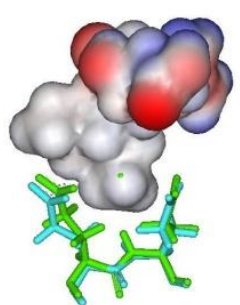

(b)

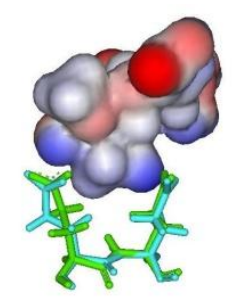

(d)
Fig. 6. E276 and E277 (stick figures) with inhibitors (electrostatic surface): a. zanamivir; b. peramivir; c. oseltamivir carboxylate; d. laninamivir.

The differences interaction of S5 subsite with different inhibitor is easily distinguished. It could only happen if there is a different partial charge in the inhibitor atoms near S5 subsite. In the Fig. $6 a, 6 b, 6 c$, and $6 d$, were captured the electrostatic surface from inhibitors while interacted with S5. It could be observed that the blue electrostatic surface, which is mean has positive charge, is only seen in zanamivir and laninamivir. This is the answer why there is different interaction happened between S5 subsite and inhibitors. It is obvious since the glutamic acid have a negative charge in its side chain which will attracts better the more positively charged region.

Accordance with Le et al. and Maki Kiso et al. studies [21], [23], our results shows that oseltamivir-resistant variants which possess a histidine-to-tyrosine substitution at position 274 (H274Y) and an asparagine-to-serine substitution at position 294 (N294S) in NA was reduced by R-125489 (laninamivir compound) and zanamivir, but not by oseltamivir carboxylate. Other oseltamivir-resistant experiment shows that oseltamivir carboxylate IC50 ratios of 32 to 8400 for H274Y H1N1, R292K, E119K, and N294S $(\mathrm{H} 3 \mathrm{~N} 2)$ mutants, whereas for laninamivir and zanamivir are 0.69 to 2.8 , and 0.72 to 1.7 , respectively [22]. The values of IC50 indicate that the ability of NA to bind both laninamivir and zanamivir is better than oseltamivir carboxylate.

\section{CONCLUSION}

This research shows a comparison of models using the combination of three different assessment methods, four inhibitors (zanamivir, laninamivir, oseltamivir carboxylate and peramivir) affinities when attached into and simulated with neuraminidase. The unique perspective of each assessment method were used in this study have its own 
advantages when being worked to selecting the best model. The preferably good structure chosen is model 3 which have the best rank in verify-3D and DOPE scores and shared the $1^{\text {st }}$ rank with model 4 . The model which was docked with four inhibitors and simulated model shows different binding affinities.The hydrogen bonds and interaction energy shows that zanamivir and laninamivir is preferable to treat a patient which infected with oseltamivir-resistant virus.

We have made two important points from this simulation study. First, the movement of oseltamivir carboxylate is relatively high compared with other inhibitors simulated and it may indicate the resistance of the virus neuraminidase. Second, the inhibitors movement and hydrogen bonds occupation comparison show that zanamivir and laninamivir are preferable for Indonesian $\mathrm{H} 274 \mathrm{Y}$ mutant. But still, further experiment with equilibrated system is needed to produce a better interaction.

\section{ACKNOWLEDGMENT}

We would like to express gratitude towards Ding Ming Chee of Accelrys Singapore for the Accelrys Discovery Studio 2.1 trial sent to us.

\section{REFERENCES}

[1] WHO. H5N1 avian influenza: Timeline of major events. 2012 [Online]. Available:

http://www.who.int/influenza/human_animal_interface/H5N1_avian influenza_update.pdf

[2] E. R. Sedyaningsih, S. Isfandari, S. Soendoro, and S. F. Supari. "Towards Mutual Trust, Transparency and Equity in Virus Sharing Mechanism: The Avian Influenza Case of Indonesia," Ann Acad Med Singapore; vol. 37, pp. 482-488, 2008.

[3] WHO, Cumulative number of confirmed human cases for avian influenza A H5N1 reported to WHO 2003-2012? March, $5^{\text {th }} 2012$ update [Online]. Available:

http://www.who.int/influenza/human_animal_interface/EN_GIP_201 20305CumulativeNumberH5N1cases.pdf

[4] T. Werner, and T. C. Harder, "Chapter 2: Avian Influenza. Influenza Report 2006, edited by Kamps et al," Flying Publisher, pp. 48-86, 2006.

[5] H. Chen, G. Deng, Z. Li, G. Tian, Y. Li, P. Jiao, L. Zhang, Z. Liu, R. G. Webster, and K. Yu. "The evolution of H5N1 influenza viruses in ducks in southern China," in Proc Natl Acad Sci U S A 101: pp. 10452-7, 2004.

[6] G. Behrens and M. Stoll, "Chapter 4: Pathogenesis and Immunology. Influenza Report 2006, edited by Kamps et al," Flying Publisher. pp. 92-109, 2006.

[7] A. C. Lowen and P. Palese, "Influenza Virus Transmission: Basic Science and Implications for the Use of Antiviral Drugs during a Pandemic," Infectious Disorders - Drug Targets, pp. 318-328, 2007.

[8] L. J. Mitnaul, M. R. Matrosovich, M. R. Castrucci, A. B. Tuzikov, N. V. Bovin, D. Kobasa, and Y. Kawaoka, "Balanced Hemagglutinin and Neuraminidase Activities Are Critical for Efficient Replication of Influenza A Virus," Journal of Virology, vol. 74, pp. 6015-6020, 2000.

[9] R. J. Russell, L. F. Haire, D. J. Stevens, P. J. Collins, Y. P. Lin, G. M. Blackburn, A. J. Hay, S. J. Gamblin, and J. J. Skehel, "The structure of H5N1 avian influenza neuraminidase suggests new opportunities for drug design," Nature, vol. 443, pp. 45-49, 2006.

[10] R. Chachra and R. C. Rizzo, "Origins of Resistance Conferred by the R292K Neuraminidase Mutation via Molecular Dynamics and Free Energy Calculations," Journal of Chemical Theory and Computation, vol. 4, pp.1526-1540, 2008.

[11] J. L. M. Breschkin, A. Sahasrabudhe, T. J. Blick, M. McDonald, P. M. Colman, G. J. Hart, R. C. Bethell, and J. N. Varghese. "Mutations in a conserved residue in the influenza virus neuraminidase active site decreases sensitivity to Neu5Ac2en derivatives," J Virol, vol. 72, pp. 2456-2462, 1998.

[12] V. P. Mishin, F. G. Hayden, and L. V. Gubareva, "Susceptibilities of Antiviral-Resistant Influenza Viruses to Novel Neuraminidase Inhibitors," Antimicrobial Agents and Chemotherapy, vol. 49, pp. 4515-4520, 2005.
[13] T. G. Sheu, V. M. Deyde, M. O. Adhiambo, R. J. Garten, X. Xu, R. A Bright, E. N. Butler, T. R. Wallis, A. I. Klimov, and L. V. Gubareva, "Surveillance for Neuraminidase Inhibitor Resistance among Human Influenza A and B Viruses Circulating Worldwide from 2004 to 2008," Antimicrobial Agents and Chemotherapy, vol. 52, pp. 3284-3292, 2008.

[14] N. T. Wetherall, T. Trivedi, J. Zeller, C. H. Savola, J. L. M. Breschkin, M. Zambon, and F. G. Hayden, "Evaluation of Neuraminidase Enzyme Assays Using Different Substrates To Measure Susceptibility of Influenza Virus Clinical Isolates to Neuraminidase Inhibitors: Report of the Neuraminidase Inhibitor Susceptibility Network," Journal of Clinical Microbiology, vol. 41, pp. 742-750, 2003.

[15] J. L. M. Breschkin, T. Trivedi, A. Hampson, A. Hay, A. Klimov, M. Tashiro, F. Hayden, and M. Zambon, "Neuraminidase Sequence Analysis and Susceptibilities of Influenza Virus Clinical Isolates to Zanamivir and Oseltamivir," Antimicrobial Agents and Chemotherapy. vol. 47: 2264-2272, 2003.

[16] H. Yen, N. A. Ilyushina, R. Salomon, E. Hoffmann, R. G. Webster, and E. A. Govorkova, "Neuraminidase Inhibitor-Resistant Recombinant A/Vietnam/1203/04 (H5N1) Influenza Viruses Retain Their Replication Efficiency and Pathogenicity in Vitro and In Vivo," Journal of Virology, vol. 81, pp. 12418-12426, 2007.

[17] A. Meijer, A. Lackenby, O. Hungnes, B. Lina, S. V. D. Werf, B. Schweiger, M. Opp, J. Paget, J. V. D. Kassteele, J. Hay, and M. Zambon, O. R. I. U. Virus A (H1N1), Europe, 2007-08 Season. Emerging Infectious Diseases, vol. 15, pp. 552-560, 2009.

[18] A. S. Monto, J. L. McKimm-Breschkin, C. Macken, A. W. Hampson, A. Hay, A. Klimov, M. Tashiro, R. G. Webster, M. Aymard, F. G. Hayden, and M. Zambon, "Detection of Influenza Viruses Resistant to Neuraminidase Inhibitors in Global Surveillance during the First 3 Years of Their Use," Antimicrobial Agents and Chemotherapy, vol. 50, pp. 2395-2402, 2006.

[19] D. Tamura, K. Mitamura, M. Yamazaki, M. Fujino, M. Nirasawa, K. Kimura, M. Kiso, H. Shimizu, C. Kawakami, S. Hiroi, S. Takahashi, M. Hata, H. Minagawa, Y. Kimura, S. Kaneda, S. Sugita, T. Horimoto, N. Sugaya, and Y. Kawaoka, "Oseltamivir-Resistant Influenza A Viruses Circulating in Japan," Journal of Clinical Microbiology, vol. 47, pp. 1424-1427, 2009.

[20] P. J. Collins, L. F. Haire, Y. P. Lin, J. Liu, R. J. Russell, P. A. Walker, J. J. Skehel, S. R. Martin, A. J. Hay, and S. J. Gamblin, "Crystal structures of oseltamivir-resistant influenza virus neuraminidase mutants," Nature, vol. 453, pp. 1258-1261, 2008.

[21] Q. M. Le, M. Kiso, K. Someya, Y. T. Sakai, T. H. Nguyen, K. H. L. Nguyen, N. D. Pham, H. H. Ngyen, S. Yamada, Y. Muramoto, T. Horimoto, A. Takada, H. Goto, T. Suzuki, Y. Suzuki, and Y. Kawaoka. Avian flu: isolation of drug-resistant H5N1 virus. Nature (London) vol. 437, pp. 1108, 2005.

[22] M. Yamashita, T. Tomozawa, M. Kakuta, A. Tokumitsu, H. Nasu, and S. Kubo, "CS-8958, a Prodrug of the New Neuraminidase Inhibitor R-125489, Shows Long-Acting Anti-Influenza Virus Activity," Antimicrobial Agents and Chemotherapy, vol. 53, no. 1, pp. 186-192, 2009.

[23] M. Kiso, S. Kubo, M. Ozawa, Q. M. Le, and C. A. Nidom, "Efficacy of the New Neuraminidase Inhibitor CS-8958 against H5N1 Influenza Viruses," PloS Pathog, vol. 6, no. 2, pp. 1-10, 2001.

[24] [Online].

Available: http://www.ncbi.nlm.nih.gov/genomes/FLU/Database/nph-select.cgi? go $=$ database

[25] S. F. Altschul, T. L. Madden, A. A. Schaffer, J. Zhang, Z. Zhang, W. Miller, and D. J. Lipman, "Gapped BLAST and PSI-BLAST: a new generation of protein database search programs," Nucleic Acids Res, vol. 25, pp. 3389-3402, 1997.

[26] E. M. Zdobnov and R. Apweiler, "InterProScan - an integration platform for the signature-recognition methods in Inter Pro," Bioinformatics, vol. 17, pp. 847-848, 2001

[27] K. Arnold, L. Bordoli, J. Kopp, and T. Schwede, "The SWISS-MODEL Workspace: A web-based environment for protein structure homology modeling," Bioinformatics, vol. 22, pp. 195-201, 2006.

[28] F. Kiefer, K. Arnold, M. Künzli, L. Bordoli, and T. Schwede, "The SWISS-MODEL Repository and associated resources," Nucleic Acids Research, vol. 37, pp. 387-392, 2009

[29] T. Schwede, J. Kopp, N. Guex, and M. C. Peitsch, "SWISS-MODEL an automated protein homology-modeling server," Nucleic Acids Research, vol. 31, pp. 3381-3385, 2003.

[30] N. Guex and M. C. Peitsch, "SWISS-MODEL and the Swiss-PdbViewer: An environment for comparative protein modeling," Electrophoresis, vol. 18, pp. 2714-2723, 1997. 
[31] M. C. Peitsch, "Protein modeling by E-mail (From amino acid sequence to protein structure: A free one-hour service)," Nature Biotechnology, vol. 13, pp. 658-660, 1995.

[32] PDB Biological Macromolecular Resource. [Online]. Available: http://www.pdb.org/pdb/home/home.do

[33] N. Saitou and M. Nei, "The neighbor-joining method: A new method for reconstructing phylogenetic trees" Mol. Biol. Evol, vol. 4, pp. 406-425, 1987.

[34] A. Sali, L. Pottertone, F. Yuan, H. V. Vlijmen, and M. Karplus, "Evaluation of comparative protein modeling by MODELLER," Proteins, vol. 23, pp. 318-326, 1995.

[35] M. Y. Shen and A. Sali, "Statistical potential for assessment and prediction of protein structures," Protein Science, vol. 15, pp. 2507-2524, 2006.

[36] R. Lüthy, J. U. Bowie, and D. Eisenberg, "Assessment of protein models with three-dimensional profiles," Nature, vol. 356, pp. 83-85, 1992.

[37] G. N. Ramachandran, C. Ramakrishnan, and V. Sasisekharan, "Stereochemistry of polypeptide chain configurations," Journal of Molecular Biology, vol. 7, pp. 95-99, 1963.

[38] W. Kabsch and C. C. Sander, "Biopolymers Dictionary of protein secondary structure: Pattern recognition of hydrogen-bonded and geometrical features," Biopolymers, vol. 22, pp. 2577-2637, 1983

[39] R. K. Fiser and A. Sali, "Modeling of loops in protein structures," Protein Science, vol. 9, pp. 1753-1773, 2000.

[40] R. A. Laskowski, M. W. MacArthur, D. S. Moss, and J. M. Thornton, "PROCHECK - a program to check the stereochemical quality of protein structures," Journal of Applied Crystallography, vol. 26, pp. 283-291, 1993.

[41] A. L. Morris, M. W. MacArthur, E. G. Hutchinson, and J. M. Thornton, "Stereochemical quality of protein structure coordinates," Proteins. Structure, Function, and Bioinformatics, vol. 12, pp. 345-364, 1992.

[42] The PubChem Project. [Online]. Available: http://pubchem.ncbi.nlm.nih.gov/

[43] G. Wu, D. H. Robertson, C. L. Brooks III, and M. Vieth, "Detailed Analysis of Grid-Based Molecular Docking: A Case Study of CDOCKER - A CHARMm-Based MD Docking Algorithm," Journal of Computational Chemistry, vol. 24, pp. 1549, 2003.

[44] B. R. Brooks, R. E. Bruccoleri, B. D. Olafson, D. J. States, S Swaminathan, and M. Karplus, CHARMM: A program for macromolecular energy minimization and dynamics calculations. Journal of Computational Chemistry, vol. 4, pp. 187-217, 1983.

[45] B. R. Brooks, C. L. Brooks III, A. D. MacKerell Jr, L. Nilsson, R. J. Petrella, B. Roux, Y. Won, G. Archontis, C. Bartels, S. Boresch, A. Caflisch, L. S. D. Caves, Q. Cui, A. R. Dinner, M. Feig, S. Fischer, J. Gao, M. Hodocek, W. Im, K. Kuczera, T. Lazaridis, J. Ma, V. Ovchinnikov, E. Paci, R. W. Pastor, C. B. Post, J. Z. Pu, M. Schaefer, B Tidor, R. M. Venable, H. L. Woodcock III, X. Wu, W. Yang, D. M.
York, and M. Karplus, CHARMM: The Biomolecular Simulation Program, Journal of Computational Chemistry, vol. 30, pp. $1545-1614,2009$.

[46] M. S. Lee, M. Feig, F. R. Salsbury Jr, and C. L. Brooks III, "New analytic approximation to the standard molecular volume definition and its application to generalized Born calculations," Journal of Computational Chemistry, vol. 24, pp. 1348-1356, 2003.

[47] J. P. Ryckaert, G. Ciccotti, and H. J. C. Berendsen. "Numerical integration of the Cartesian equations of motion of a system with constraints: molecular dynamics of n-alkanes," Journal of Computational Physics, vol. 23, pp. 327-341, 1977.

[48] C. L. Brooks III, B. M. Pettitt, and M. Karplus, "Structural and energetic effects of truncating long ranged interactions in ionic and polar fluids," Journal of Chemical Physics, vol. 83, pp. 5897-5908, 1985.

[49] P. J. Steinbach and B. R. Brooks, "New spherical-cutoff methods for long-range forces in macromolecular simulation," Journal of Computational Chemistry, vol. 15, pp. 667-683, 1994.

[50] A. R. Leach, "Molecular Modelling: Principles and Applications". $2^{\text {nd }}$ Edition. Prentice Hall, pp. 165-181, 2001.

[51] P. Decha, T. Rungrotmongkol, P. Intharathep, M. Malaisree, O. Aruksakunwong, C. Laohpongspaisan, V. Parasuk, P. Sompornpisut, S Pianwanit, S. Kokpol, and S. Hannongbua, "Source of High Pathogenicity of an Avian Influenza Virus H5N1: Why H5 Is Better Cleaved by Furin," Biophysical Journal, vol. 95, pp. 128-134, 2008.

[52] X. L. Guo, D. Q. Wei, Y. S. Zhul, and K. C. Chou, "Cleavage mechanism of the H5N1 hemagglutinin by trypsin and furin," Amino Acids, vol. 35, pp. 375-382, 2008.

[53] M. Shu, Z. Lin, Y. Zhang, Y. Wu, H. Mei, and Y. Jiang, "Molecular dynamics simulation of oseltamivir resistance in neuraminidase of avian influennza H5N1 virus," J Mol Model, 2010.

[54] V. Stoll, K.D. Stewart, C.J. Maring, S. Muchmore, V. Giranda, Y.-g.Y. Gu, G. Wang, Y. Chen, M. Sun, C. Zhao, A.L. Kennedy, D. L Madigan, Y. Xu, A. Saldivar, W. Kati, G. Laver, T. Sowin, H. L. Sham, J. Greer, and D. Kempf, "Influenza Neuraminidase Inhibitors: Structure-Based Design of a Novel Inhibitor Series," Biochemistry, vol. 42, pp. 718-727, 2003.

[55] O. Aruksakunwong, M. Malisree, P. Decha, P. Sompornpisut, V. Parasuk, S. Pianwanit, and S. Hannongbua, "On the Lower Susceptibility of Oseltamivir to Influenza Neuraminidase Subtype than Those in N2 and N9," Biophysical Journal, vol. 92, pp. 798-807, 2007.

[56] L. Le, E. Lee, K. Schulten, and T.N. Truong, "Molecular Modeling of swine influenza A/H1N1, Spanish H1N1, and avian H5N1 flu N1 Neuraminidase bound to Tamiflu and Relenza," PloS Current Beta, 2009.

[57] J. Gong, W. Xu, and J. Zhang, "Structure and Functions of Influenza Virus Neuraminidase," Current Medicinal Chemistry, vol. 14, pp. 113-122, 2007. 